\title{
ENVIRONMENTAL EFFECTS IN THE EVOLUTION OF GALACTIC BULGES
}

\author{
Lauren A. Macarthur ${ }^{1,2,3}$, Richard S. Ellis ${ }^{3}$, Tommaso Treu ${ }^{4}$, and Sean M. Moran ${ }^{5}$ \\ ${ }^{1}$ Herzberg Institute of Astrophysics, National Research Council of Canada, 5071 West Saanich Road, Victoria, BC V8X 4M6, Canada; \\ Lauren.MacArthur@nrc-cnrc.gc.ca \\ 2 Department of Physics \& Astronomy, University of Victoria, Victoria, BC, V8P 1A1, Canada \\ ${ }^{3}$ Department of Astrophysics, California Institute of Technology, MS 105-24, Pasadena, CA 91125, USA; rse@astro.caltech.edu \\ ${ }^{4}$ Department of Physics, University of California, Santa Barbara, CA 93106-9530, USA; tt@physics.ucsb.edu \\ ${ }^{5}$ Department of Physics \& Astronomy, The Johns Hopkins University, Baltimore, MD 21218, USA; moran@ pha.jhu.edu \\ Received 2009 July 3; accepted 2009 December 7; published 2010 January 4
}

\begin{abstract}
We investigate possible environmental trends in the evolution of galactic bulges over the redshift range $0<z<0.6$. For this purpose, we construct the fundamental plane (FP) for cluster and field samples at redshifts $\langle z\rangle=0.4$ and $\langle z\rangle=0.54$ using surface photometry based on Hubble Space Telescope imaging and velocity dispersions based on Keck spectroscopy. As a reference point for our study, we include data for pure ellipticals, which we model as single-component Sérsic profiles; whereas for multi-component galaxies we undertake decompositions using Sérsic and exponential models for the bulge and disk, respectively. Although the FP for both distant cluster and field samples is offset from the local relation, consistent with evolutionary trends found in earlier studies, we detect significant differences in the zero point of $\simeq 0.2$ dex between the field and cluster samples at a given redshift. For both clusters, the environmentally dependent offset is in the sense expected for an accelerated evolution of bulges in dense environments. By matching the mass range of our samples, we confirm that this difference does not arise as a result of the mass-dependent downsizing effects seen in larger field samples. Our result is also consistent with the hypothesis that-at fixed mass and environment- the star formation histories of galactic bulges and pure spheroids are indistinguishable and difficult to reconcile with the picture whereby the majority of large bulges form primarily via secular processes within spiral galaxies.
\end{abstract}

Key words: galaxies: evolution - galaxies: formation - galaxies: spiral - galaxies: stellar content

\section{INTRODUCTION}

A spheroid can exist as an independent structure, i.e., a pure elliptical galaxy, or it can be surrounded by a disk component, i.e., the bulges of spiral and lenticular galaxies. Good progress has been achieved in the past decade in constraining the past star formation history of spheroid-dominated E/SO galaxies through studies of the redshift-dependent fundamental plane (FP) - the relation between galaxy size, surface brightness, and stellar velocity dispersion (e.g., Treu et al. 2002, 2005; van Dokkum \& van der Marel 2007). More recently, these analyses have been extended to include the bulges of spiral galaxies (MacArthur et al. 2008, hereafter Mac08; Hathi et al. 2009), allowing a direct comparison between the mass assembly history of pure spheroids and those residing in disks. This enables us to test the simple conjecture that galactic bulges share a similar assembly history to isolated spheroids and are not, at least to first order, significantly influenced in their growth by disk-related processes. While the bulge samples remain small and can only currently be compiled to redshifts $z<1$, the general picture that has emerged is that both isolated spheroids and galactic bulges evolved at a similar rate for a given spheroid mass and with similar mass-dependent trends. In particular, for both systems the less massive examples have witnessed more recent activity whereas the most massive ones formed the vast majority of their stars at high redshift $\left(z_{f} \gtrsim 2\right)$.

A key question of interest for both bulges and isolated spheroids is the role of the local environment. While the influence of the local environment on the morphological mix (Dressler 1980; Smith et al. 2005), colors (Bamford et al. 2009), mass-to-light ratios (Moran et al. 2005; hereafter M05), and star formation rates (Lewis et al. 2002; Kauffmann et al. 2004; Cooper et al. 2008) of galaxies is now well established, some studies have emphasized the importance of mass as the governing evolutionary factor (e.g., Treu et al. 2005; Mac08). Separating the effects of mass from that of environmental density is thus clearly important. Environmental trends are qualitatively consistent with the predictions of popular structure formation models where growth is accelerated in more massive halos which start their collapse at earlier times (Governato et al. 2007). By contrast, mass-dependent trends require feedback processes which are poorly understood.

For the E/S0 population, the question of the relative importance of the environment is still somewhat open. In contrast to field versus cluster trends found in early work (Treu et al. 2002), van Dokkum \& van der Marel (2007) found only marginal differences in the inferred ages for massive galaxies as a function of their environment. However, M05, studying E/S0s spanning a wide range of cluster-centric distance within a single cluster, $\mathrm{Cl} 0024+17(z=0.4)$, found a significant radial trend. This is in the sense of a decreasing $M / L$ ratio with both cluster-centric distance and local density, so that early types close to the cluster core have older ages, while those at the periphery are younger, and more similar to field galaxies at a similar redshift. A key concern in attempting to reconcile these various results is the mass range explored in the various samples, as is known that the mass function depends on the environment.

Clearly, it is desirable to characterize the past history of bulges and spheroidal galaxies as a function of both mass and environment. Recognizing this, we extend the scope of our earlier work (Mac08) and present here a comparative field versus cluster FP analysis in two redshift bins defined by the clusters $\mathrm{Cl} 0024+17(z=0.4)$ and MS 0451-03 $(z=0.54)$. FP parameters are derived from Keck/DEIMOS spectroscopy to measure velocity dispersions and Hubble Space Telescope (HST) imaging for photometric parameters, supplemented with 
ground-based Canada-France-Hawaii Telescope (CFHT) and SUBARU imaging. For all distance-dependent quantities we adopt a flat cosmological model with $\Omega_{M}=0.3, \Omega_{\Lambda}=0.7$, and $\mathrm{H}_{0}=65 \mathrm{~km} \mathrm{~s}^{-1} \mathrm{Mpc}^{-1}$. All magnitudes are in the AB system (Oke 1974).

\section{DATA}

Imaging data for the field samples are largely taken from the GOODS survey (Giavalisco et al. 2004) which provides deep imaging in four HST-ACS passbands. The broad wavelength coverage permits the derivation of accurate $k$-corrections for the selected galaxies, which all have measured spectroscopic redshifts from the Keck Team Redshift Survey (Wirth et al. 2004). Additional field galaxies were included from the sample of spectroscopically confirmed non-members located within the two cluster fields (see Moran et al. 2007b).

Imaging data for the cluster samples is available from previous HST campaigns described fully in Moran et al. (2007a; hereafter M07a). Both clusters were observed in the F814W filter with WFPC2 (for Cl 0024+17) and ACS (for MS 0451-03). Galaxies were selected from the comprehensive spectroscopic survey described by M07a. Accurate $k$-corrections were derived using optical $(B V R I)$ ground-based imaging from the CFHT and SUBARU telescopes. Due to the inferior resolution of the ground-based data, aperture magnitudes of radius 0.' 6 were used for the $k$-corrections. Previous studies have shown (Ellis et al. 2001; Mac08) that disk contamination is minimal within such an aperture.

Both field and cluster galaxies were selected morphologically via visual inspection to a limit of $i_{\mathrm{AB}} \sim 21.5$. Details of the classification and its reliability are presented in Treu et al. (2003) and Bundy et al. (2005) for the cluster and field samples, respectively.

The Keck spectroscopic data arise from a number of independent campaigns. The bulk of the velocity dispersions in the cluster fields derives from a $i_{\mathrm{AB}}<21.5$ sample discussed by M05/M07a. This sample was augmented via an additional observing run in November 2007 dedicated to increasing the cluster samples for this purpose. Here, we selected further cluster galaxies with $i_{\mathrm{AB}}<21.5$ and a bulge/total $(B / T)$ fraction $>0.2$ determined from decompositions of the HST imaging. The opportunity also enabled us to also enlarge the observed sample in the GOODS-S field with respect to the GOODS field samples presented in Mac08. Typical exposure times on this latest run were 5-6 hr with seeing conditions in the range $0.6-1$.'. 1 .

To facilitate the desired comparison between field and cluster, we defined the GOODS-N/S field samples to be those in the above compilation within the redshift ranges $0.3<z \leqslant 0.5$ (for comparison with the $\mathrm{Cl0024+17}$ sample) and $0.5<z \leqslant 0.7$ (for comparison with the MS0451-03 sample). Nine field galaxies were accepted from the clustered fields lying within these relevant boundaries and $4 \sigma$ outside the cluster redshift distribution.

The resulting sample comprises 240 spheroids (133 clusters, 107 fields) of which 175 (94 clusters, 81 fields) represent bulges in two-component galaxies and 65 (39 clusters, 26 fields) represent single-component spheroidal galaxies.

\section{ANALYSIS}

The derivation of FP parameters from the spectroscopic and photometric data discussed above closely followed the procedures discussed in detail in Mac08 to which the reader is referred. Briefly, size and surface brightness parameters were determined from one-dimensional profiles (see MacArthur et al. 2003 for details) following decomposition of the HST images into Sérsic bulge and exponential disk components. For pure elliptical galaxies, only a single Sérsic profile was fit. Stellar velocity dispersions were measured from the Keck/DEIMOS spectra using the Gauss-Hermite pixel Fitting algorithm (van der Marel 1994). By limiting the sample to systems with $B / T>0.2$, contamination from the disk to the central velocity dispersion measurement is insignificant (Mac08).

While all spectroscopic data sets were obtained with Keck/ DEIMOS, there were a few differences in the observational setup and measurement procedures across the various cluster subsamples which could affect the field-cluster comparison. These differences reflect the fact that the present bulge comparison was not envisaged when the M05/M07a cluster study was planned (and do not apply to the field samples of Mac08). Two differences are worth considering.

Firstly, the bulk of the early cluster galaxy spectra was taken over restframe $3500-6700 \AA$ with a $6001 \mathrm{~mm}^{-1}$ grating suitable for E/S0 galaxies, whereas the 2007 November data sampled restframe $3600-5400 \AA$ with a $12001 \mathrm{~mm}^{-1}$ grating appropriate for less massive systems. In probing a mixed stellar population, a redder wavelength range might be more sensitive to older stars.

A second difference arises in the extraction of the spectra. All data sets were reduced using the DEEP2 pipeline (Davis et al. 2003) which extracts both a one-dimensional optimally weighted spectrum and a two-dimensional spectrum. The M05/ M07a analyses used the one-dimensional spectrum to derive dispersions whereas, for the bulges, only the central bin was used with no co-addition of pixels. The latter data thus have a fixed effective aperture of 0.35 , while the Moran et al. data have a larger and slightly variable aperture depending on how many pixels were coadded for the one-dimensional spectrum. Conceivably these aperture differences could lead to subtle biases in our desired comparison.

Fortunately, repeat observations of a number of galaxies between the two samples allows us to constrain this possible bias. For $\mathrm{Cl} 0024+17$, after correcting to a common effective aperture of radius $r_{\mathrm{e}} / 8$, there is no significant average offset for the 19 galaxies common to both data sets $0.01 \pm 0.02$ dex. However, for MS 0451-03, after aperture correction, a difference of $0.08 \pm 0.02$ dex toward larger dispersions for the Moran et al. measurements was found. Although still only a small effect compared to the final differences that we measure between cluster and field, to verify the source of this offset we reanalyzed the Moran et al. data using exactly the technique adopted for the new data. We found that the small offset can, in fact, be attributed to the different wavelength range. In the re-analysis of the Moran et al. data the red end was masked during the fit, producing consistent results. We conclude that the effect is due to the presence of composite stellar populations and can be mitigated by focusing on the same exact wavelength region. Thus, to correct the Moran et al. dispersions in MS 0451-03 to the same scale as the other data, a shift of 0.08 dex was added to them for the current analysis. We stress that this in no way invalidates the earlier discussions of these data which were internally consistent.

Finally, in order to measure the evolutionary trends, a suitable local reference FP is required. For this we use the relation of Jørgensen et al. (1996, hereafter J96) for early-type Coma cluster galaxies. This choice does not provide an ideal comparison 


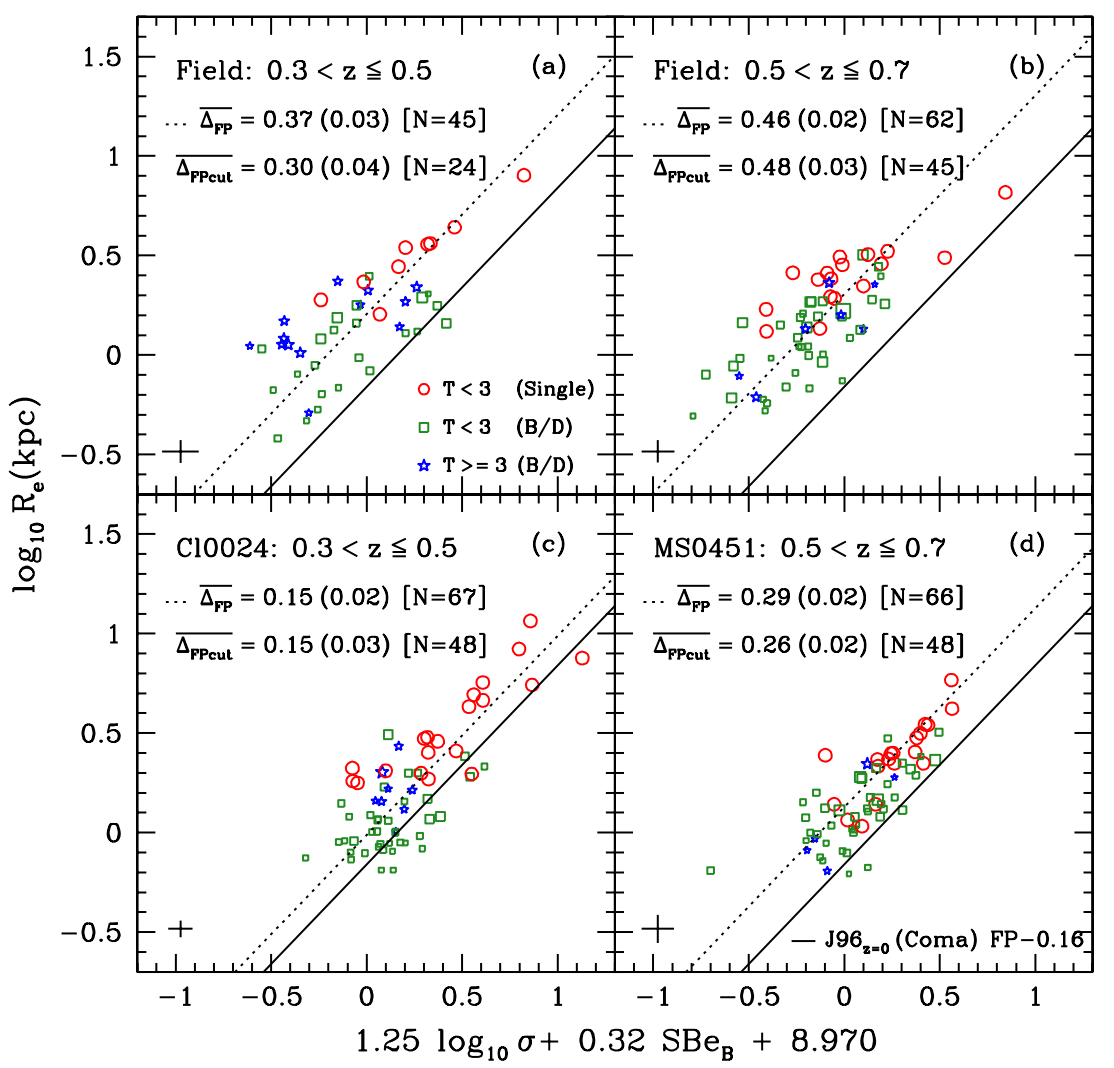

Figure 1. Fundamental plane (FP) for field (upper panels) and cluster (lower panels) samples in two redshift bins. Point types and colors are as follows: red circles: single-component spheroids (i.e., "pure" ellipticals), green squares: bulges of two-component systems with T-type $<3(\equiv S a+b)$, and blue stars: bulges of twocomponent systems with T-type $\geqslant 3$ and $B / T>0.2$. The solid lines are the local J96 relation for Coma corrected as discussed in the text. Dotted lines represent the mean offset from the local FP for each sub-sample. The mean offset, $\overline{\Delta_{\mathrm{FP}}}$, error on the mean and the number of galaxies are indicated at the top left of each panel. The corresponding numbers are also given for the mass-limited sub-samples $\left(\overline{\Delta_{\text {FPcut }}}\right.$, see the text).

for the current study as it does not account for structural nonhomology or any disk component in their sample galaxies. The effects of this difference were explored in Mac08, revealing an offset (equivalent to $0.16 \mathrm{dex}$ in the local zero point) in the structural parameters from the fixed $n=4$ single Sérsic fits versus the best-fit Sérsic $n$ having also fit a disk component when present. Thus, for a direct comparison with our higher- $z$ samples, we adopt the shifted J96 FP as our local zero point.

\section{RESULTS}

Having established homogeneous data sets of field and cluster spheroids in two redshift bins and a suitable local comparison relation, we make a direct differential comparison of the FP in each bin. In Figure 1, we plot the FP for our sample separated into two redshift bins for the field (top) and cluster (bottom) samples. The solid line marks the local relation. The comparison of the field versus cluster samples shows a similar difference for the spheroidal FP zero points of $0.22 \pm 0.04$ dex and $0.17 \pm 0.03 \mathrm{dex}$ for the successive redshift bins, in the sense that the cluster spheroidals are systematically fainter at fixed size and velocity dispersion, consistent with older stellar populations.

The key bias that could affect the intended analysis is the mass range sampled in each redshift bin. Given our earlier discussion, to isolate environmental effects from those arising as a result of mass-dependent effects (Bundy et al. 2005; Treu et al. 2005; van der Wel et al. 2005; Mac08), it is important to consider carefully the mass range for field and cluster spheroids. We determine the dynamical mass using the relation $M \equiv k(n) \sigma^{2} R_{e} / G$, where $k(n)$ is a profile shape-dependent virial coefficient taken from Trujillo et al. (2004). Although such a derived mass is affected by the contribution of the disk and dark matter components, so long as we undertake a differential comparison, we consider this a minor effect.

Figure 2 shows the dependence of the FP offset derived from Figure 1 as a function of spheroid mass. Here, we see that the field samples are indeed more heavily weighted toward lower masses and also show evidence of the downsizing trends discussed, e.g., by Treu et al. (2005). To determine rigorously whether there is an additional environmental trend, we thus recompute the FP offsets restricting both cluster and field samples to lie within the mass interval $\log \left(M / M_{\odot}\right)=10.5-11.6$ (chosen such that both datasets adequately sample the range) . $^{6}$. The new offsets are shown in Figure 1 as " $\Delta_{\text {FPcut }}$." With these refined samples, the new field versus cluster FP zero point shifts become $0.15 \pm 0.05$ dex and $0.22 \pm 0.04$ dex for the successive redshift bins. Further restriction on the mass range such that the sampling in the field and cluster bins are matched also does not change the results. In summary, there is a clear environmental effect even when the mass and $z$ ranges of the field and cluster spheroid samples are restricted to be the same (i.e., the trend is not driven by selection effects).

\footnotetext{
6 A Kolmogorov-Smirnov test confirms that the mass distribution is not significantly different for the low- $z$ bin, and examination of selection limits as in Figure 11 in Mac08 confirms that no galaxies in the low- $z$ bin would have been missed in the high- $z$ bin. Additionally, restricting the field bin to as narrow as $0.53<z \leqslant 0.56$ again does not alter our results.
} 


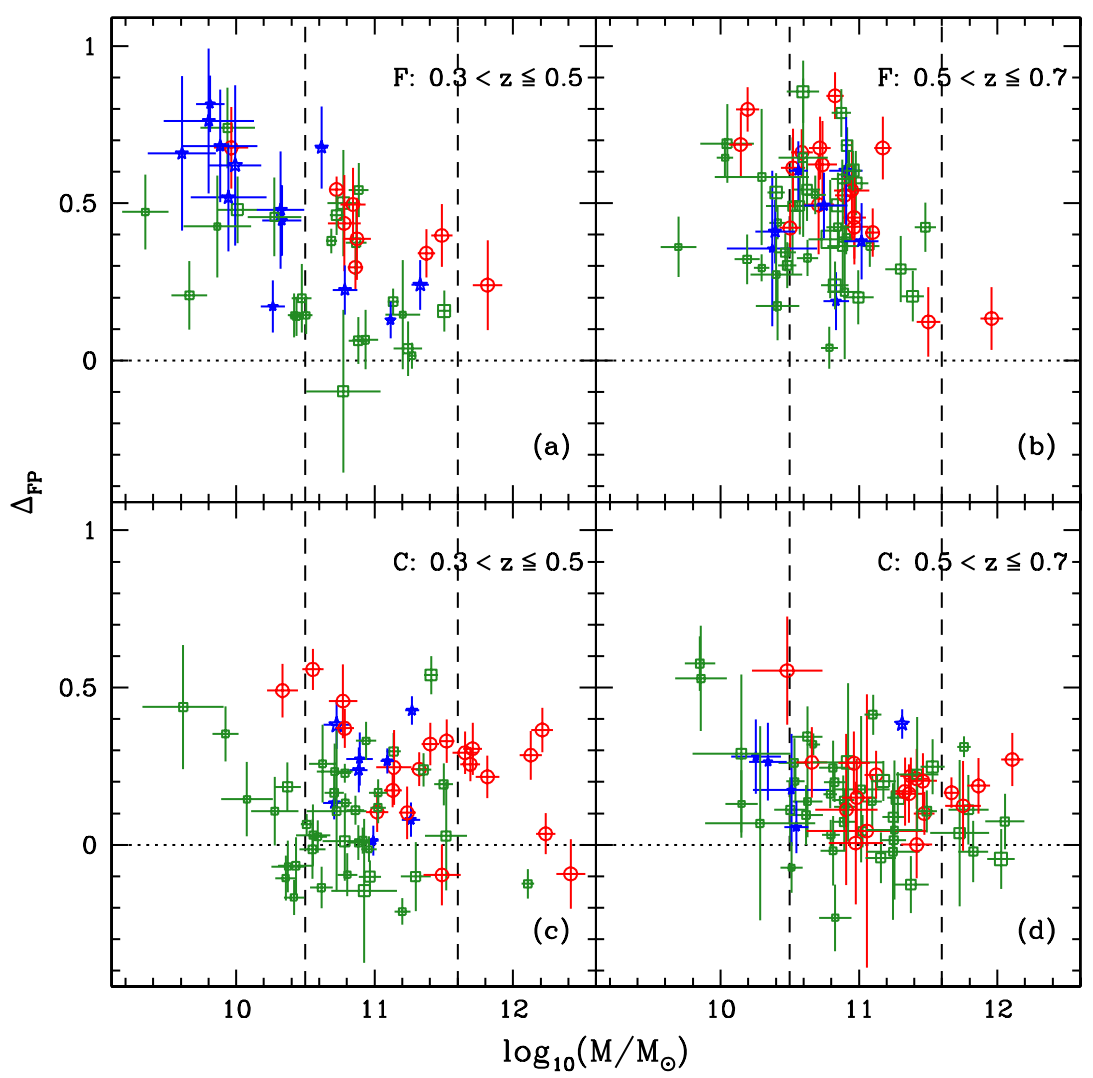

Figure 2. Offset from the local FP relation as a function of dynamical spheroid mass. Point types and colors are the same as in Figure 1. The dashed vertical lines indicate the mass limits appropriate for the restricted sample for the $\Delta_{\text {FPcut }}\left(10.5 \leqslant \log \left(M / M_{\odot}\right) \leqslant 11.6\right)$ values in Figure 1.

\section{DISCUSSION}

Figure 3 summarizes the primary result of this Letter. We observe a strong environmental signal in both redshift bins for all spheroids with $B / T>0.2$. This signal is not a manifestation of mass-dependent trends and thus is in addition to the downsizing trends observed before. In terms of star formation history, the observed trends can be interpreted as follows. In the cluster environment, the spheroidal component of galaxies is consistent with having formed very few stars below redshift $z \sim 2$, in agreement with the general trend observed for pure spheroidals of the same mass. In contrast, in the field environment, the evolution of the FP is significantly faster, consistent with a more recent stellar population (a single star formation episode would imply formation redshifts of $z \simeq 0.8$ ). However-as in the case of pure spheroidals (e.g., Treu et al. 2005) - a more likely interpretation of the observed trends is that the majority of the stars are formed at significantly higher redshift, and the integrated stellar populations are then rejuvenated by secondary episodes of star formation below $z \sim 1$. As an illustration, we show the expected evolution for a model where $15 \%$ of the stellar mass is formed at $z=0.62$. The similarity of the star formation histories of galactic bulges and pure spheroids appears difficult to reconcile with the picture whereby the majority of large bulges form primarily via secular processes within spiral galaxies.

In the case of pure spheroidals of high mass, a decisive argument in favor of the secondary bursts model is the relatively slow evolution of their stellar mass function since $z \sim 1$ (Bundy et al. 2005). Unfortunately, no such measurement is currently available for the spheroid and stellar components of galaxies since $z \sim 1$ to break the degeneracy between star

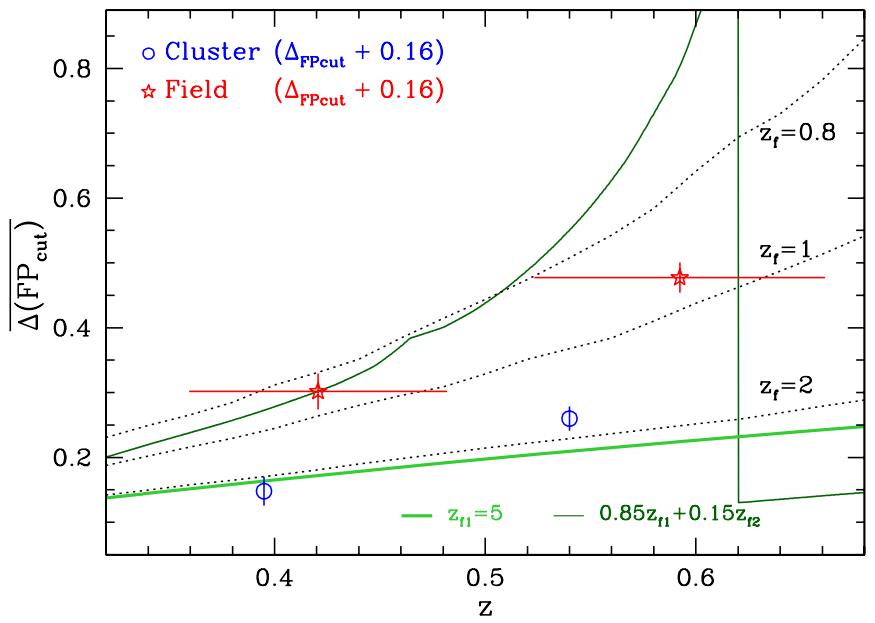

Figure 3. Average offset from the local FP relation as a function of redshift for the mass-restricted sample for field (red stars) and cluster (open circles) samples. The points for the field sample are located at the mean $z$ of the sample, and the horizontal bars represent the rms spread. Dotted black lines represent model tracks of a passively evolving single burst population of solar metallicity for three formation redshifts $\left(z_{f}=0.8,1,2\right)$ from the models of Bruzual \& Charlot (2003). The solid lines represent an initial burst of star formation at $z_{f 1}=5$ (light green) with a second burst comprising $15 \%$ of the stars by mass added at $z_{f 2}=0.62$ (dark green).

formation history and assembly history. The recent growth of the spheroidal component of lenticular galaxies (e.g., Geach et al. 2009) and the observed evolution of the morphology density relation (e.g., Smith et al. 2005) indicate that the pure luminosity evolution is not likely to be an appropriate model for the evolution of the spheroidal component, although at the moment there is not enough information to disentangle the dependency 
of the demographics of spheroids on mass and environment. A measurement of the evolution of the mass function of bulges and disks as a function of environment is needed to make further progress.

L.A.M. acknowledges financial support from the National Science and Engineering Council of Canada. T.T. acknowledges support from the NSF through CAREER award NSF-0642621, by the Sloan Foundation and by the Packard Foundation. R.S.E. acknowledges financial support from the Royal Society. Some of the data presented herein were obtained at the W. M. Keck Observatory, which is operated as a scientific partnership among the California Institute of Technology, the University of California and the National Aeronautics and Space Administration. The Observatory was made possible by the generous financial support of the W. M. Keck Foundation. This work is partly based on archival data from the Hubble Space Telescope, obtained from the data archive at the Space Telescope Institute, which is operated by the association of Universities for Research in Astronomy, Inc. for NASA under contract NAS5-26555.

\section{REFERENCES}

Bamford, S. P., et al. 2009, MNRAS, 393, 1324

Bruzual, A. G., \& Charlot, S. 2003, MNRAS, 344, 1000 [BC03]

Bundy, K., Ellis, R. S., \& Conselice, C. J. 2005, ApJ, 625, 621

Cooper, M. C., et al. 2008, MNRAS, 383, 1058

Davis, M., et al. 2003, Proc. SPIE, 4834, 161

Dressler, A. 1980, ApJ, 236, 351
Ellis, R. S., Abraham, R. G., \& Dickinson, M. 2001, ApJ, 551, 111

Geach, J. E., Smail, I., Moran, S. M., Treu, T., \& Ellis, R. S. 2009, ApJ, 691, 783

Giavalisco, M., et al. 2004, ApJ, 600, L93

Governato, F., Willman, B., Mayer, L., Brooks, A., Stinson, G., Valenzuela, O., Wadsley, J., \& Quinn, T. 2007, MNRAS, 374, 1479

Hathi, N. P., Ferreras, I., Pasquali, A., Malhotra, S., Rhoads, J. E., Pirzkal, N., Windhorst, R. A., \& Xu, C. 2009, ApJ, 690, 1866

Jørgensen, I., Franx, M., \& Kjaergaard, P. 1996, MNRAS, 280, 167 [J96]

Kauffmann, G., White, S. D. M., Heckman, T. M., Ménard, B., Brinchmann, J., Charlot, S., Tremonti, C., \& Brinkmann, J. 2004, MNRAS, 353, 713

Lewis, I., et al. 2002, MNRAS, 334, 673

MacArthur, L. A., Courteau, S., \& Holtzman, J. A. 2003, ApJ, 582, 689

MacArthur, L. A., Ellis, R. S., Treu, T., U, V., Bundy, K., \& Moran, S. 2008, ApJ, 680, 70 [Mac08]

Moran, S. M., Ellis, R. S., Treu, T., Smail, I., Dressler, A., Coil, A. L., \& Smith, G. P. 2005, ApJ, 634, 977 [M05]

Moran, S. M., Ellis, R. S., Treu, T., Smith, G. P., Rich, R. M., \& Smail, I. 2007a, ApJ, 671, 1503 [M07a]

Moran, S. M., Miller, N., Treu, T., Ellis, R. S., \& Smith, G. P. 2007b, ApJ, 659, 1138

Oke, J. B. 1974, ApJS, 27, 21

Smith, G. P., Treu, T., Ellis, R. S., Moran, S. M., \& Dressler, A. 2005, ApJ, 620, 78

Treu, T., Ellis, R. S., Kneib, J.-P., Dressler, A., Smail, I., Czoske, O., Oemler, A., \& Natarajan, P. 2003, ApJ, 591, 53

Treu, T., Stiavelli, M., Casertano, S., Møller, P., \& Bertin, G. 2002, ApJ, 564, L13

Treu, T., et al. 2005, ApJ, 633, 174

Trujillo, I., Burkert, A., \& Bell, E. F. 2004, ApJ, 600, L39

van der Marel, R. P. 1994, MNRAS, 270, 271

van der Wel, A., Franx, M., van Dokkum, P. G., Rix, H.-W., Illingworth, G. D., \& Rosati, P. 2005, ApJ, 631, 145

van Dokkum, P. G., \& van der Marel, R. P. 2007, ApJ, 655, 30

Wirth, G. D., et al. 2004, AJ, 127, 3121 